\title{
ANÁLISE DE ROBUSTEZ DE ESTAÇÕES DA REDE GNSS-SP
}

\author{
Robustness analysis of stations from the GNSS-SP network. \\ EMERSON PEREIRA CAVALHERI \\ JOÃO CARLOS CHAVES \\ Universidade Estadual Paulista - UNESP \\ Programa de Pós-Graduação em Ciências Cartográficas \\ Departamento de Cartografia \\ Rua Roberto Simonsen, 305 \\ CEP 19060-900 - Presidente Prudente - SP - Brasil \\ ep.cavalheri@gmail.com; jcchaves@fct.unesp.br.
}

\section{RESUMO}

Atualmente, a necessidade de coordenadas confiáveis tem sido um dos objetivos da comunidade científica e prática. Desta forma, a análise de robustez de uma rede geodésica, tem como objetivos, com base nos erros máximos não detectados, analisar se a rede é "robusta" ou não. A rede será robusta se a influência destes erros for pequena, caso contrário é "fraca", ou seja, "não robusta". Esta análise se faz com a fusão de duas técnicas, uma que trata da análise estatística de confiabilidade e outra sobre a análise geométrica de deformações. A análise de confiabilidade fornecerá o erro máximo que não pode ser detectado por testes, após o ajustamento. Depois de encontrar estes erros, a análise geométrica de deformações determinará o potencial de deformação que esta rede terá com base nestes erros. Ressalta-se, ainda, que a análise de robustez não é dependente de datum, refletindo somente na geometria da rede e na acurácia das observações (VANİCEK et al., 2001). Portanto, este trabalho tem como propósito contribuir com as investigações científicas sobre redes geodésicas, checando a mesma, com base em sua geometria e observações.

Palavras-chave: Robustez; Confiabilidade; Deformação.

\section{ABSTRACT}

Currently, the need of reliable coordinates has been one of the main objectives of the scientific and practice community. Thus, the robustness analysis of a geodetic network, aims, at analyzing if the network is "robust" or not, based on the maximum undetectable errors. The network will be robust if the influence of these errors is 
small, otherwise it is "weak", or "not robust". This analysis is performed with the merger of two techniques, one which deals with the statistical analysis of reliability and the other one with the geometric strength analysis. The reliability analysis will provide the maximum error that cannot be detected by tests, after the adjustment. After finding these errors, the geometric strength analysis will determine the potential strain that the network will have, based on these errors. It is emphasized that the robustness analysis doesn't depend of the datum, reflecting only the geometry of the network and the accuracy of the observations (VANİCEK et al., 2001). Therefore, this work aims at contributing to the scientific research on geodetic networks, checking the same, based on their geometry and observations.

Keywords: Robustness; Reliability; Strain.

\section{INTRODUÇÃO}

No estabelecimento de redes geodésicas, as coordenadas dos pontos são estimadas usando o MMQ (Método dos Mínimos Quadrados). Para isso, as observações devem estar isentas de erros, pois se estes existirem e não forem corrigidos, serão associados diretamente aos parâmetros. Portanto, estes erros devem ser encontrados e corrigidos e, posteriormente, a rede deve ser reajustada (BERBER, 2006). Geralmente, o teste estatístico (data-snooping) utilizado para detectar erros é o proposto por Baarda (1968). Pioneiro em considerar a possibilidade do teste não conseguir detectar os erros, Baarda formulou a teoria de confiabilidade, onde determina a magnitude de um erro grosseiro que não pode ser detectado, a um nível de probabilidade $\alpha_{0}$ quando aceito a um nível de risco $\beta_{0}$, ou seja, de cometer um erro do tipo II, aceitar que não existam erros grosseiros, quando há a presença dos mesmos (VANİCEK et al., 2001).

Definida a teoria de confiabilidade e associando-se a ela a análise geométrica de deformação, tem-se a técnica de análise de robustez. Vanìcek et al. (2001) mostram que a análise tradicional de confiabilidade tem sido ampliada com a análise geométrica de rigidez (strength) usando técnicas de deformações (strains), resultando em uma extensão da teoria da confiabilidade chamada de análise de robustez. A análise de robustez de uma rede geodésica pode ser entendida como a combinação de técnicas estatísticas e de análises geométrica de deformações, na qual permite obter informações a respeito do potencial de deformação desta rede. Este potencial trata de obter informações das deformações sofridas pela rede, em decorrência dos erros mínimos nas observações e a certo nível de confiança. Com o propósito de visualizar, em termos geométricos, a análise geométrica de deformações, propõe-se um critério de rigidez, que consiste em analisar, em valores de tolerância, as deformações sofridas pelas análises de confiabilidade. A técnica de medida de robustez reflete apenas a geometria da rede e a acurácia das observações. Contudo, para calcular o deslocamento causado pelo erro máximo não detectável, condições iniciais devem ser determinadas. Além disso, valores limites são necessários para avaliar a rede, os quais são dados para determinar a robustez da 
rede. Se os deslocamentos individuais dos pontos da rede são maiores que os valores limites, deve-se alterar a geometria da rede, ou, melhorar as observações, até obter uma rede com robustez aceitável.

Portanto, este trabalho analisa a robustez de estações que pertencem à rede GNSS-SP, situadas especificamente no oeste do estado de São Paulo. Os dados GNSS (Global Navigation Satelite System) no formato RINEX (Receiver INdependent EXchange) das estações estão disponíveis no IBGE (Instituto Brasileiro de Geografia e Estatística) em: <ftp://geoftp.ibge.gov.br/RBMC/>, e são processados utilizando o software científico GAMIT/GLOBK, desenvolvidos pelo MIT (Massachusetts Institute of Technology). Após os processamentos, obtêm-se as linhas de base ajustadas da rede e, realizando o ajustamento pelo MMQ determinam-se as coordenadas das estações. A análise da robustez da rede se dá posteriormente, na comparação de um deslocamento, obtido em função das análises de confiabilidade e deformações, com os limiares de aceitação, que são obtidos em função da acurácia da rede.

\section{CONFIGURAÇÃO GEOMÉTRICA DA REDE}

Neste trabalho, utilizam-se das seguintes estações da rede GNSS-SP: PPTE, ROSA, OURI, ILHA, SPAR e SJRP, bem como a estação UFPR, situada no estado do Paraná. As estações SJRP e UFPR foram consideradas estáveis nos processamentos.

Considerando as estratégias de processamentos dos dados GNSS, baseadas em Herring et al. (2010), geram-se as soluções com os programas GAMIT/GLOBK. Na Figura 1, ilustra-se a geometria de algumas linhas de base da rede investigada e suas estações.

Figura 1 - Configuração geométrica e estações da rede.

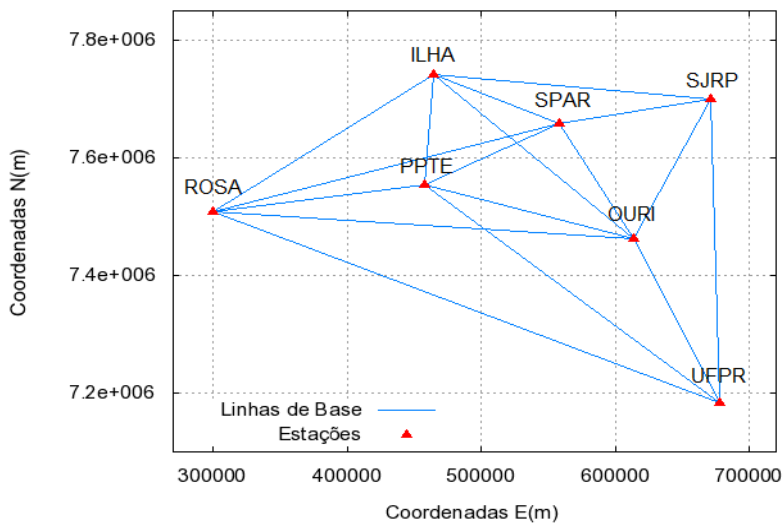

Bol. Ciênc. Geod., sec. Artigos, Curitiba, v. 20, no 2, p.376-387, abr-jun, 2014. 
As coordenadas cartesianas das estações, e seus respectivos desvios padrão estão na Tabela 1 .

Tabela 1 - Coordenadas cartesianas e desvio padrão das estações investigadas.

\begin{tabular}{l|l|l}
\hline \multirow{2}{*}{ Rede relativa } & $\begin{array}{l}\text { Coordenadas } \\
\text { cartesianas }(\boldsymbol{m})\end{array}$ & Desvio padrão $(\boldsymbol{m})$ \\
\hline \multirow{4}{*}{ PPTE } & $\mathrm{X}=3687624,3853$ & $\sigma_{X}^{\text {PPTE }}=0,0026$ \\
\cline { 2 - 3 } & $\mathrm{Y}=-4620818,7217$ & $\sigma_{Y}^{P P T E}=0,0030$ \\
\cline { 2 - 3 } & $\mathrm{Z}=-2386880,2578$ & $\sigma_{Z}^{\text {PPTE }}=0,0018$ \\
\hline \multirow{4}{*}{ OUSA } & $\mathrm{X}=3551520,5051$ & $\sigma_{X}^{\text {ROSA }}=0,0025$ \\
\cline { 2 - 3 } & $\mathrm{Y}=-4704836,1572$ & $\sigma_{Y}^{\text {ROSA }}=0,0030$ \\
\cline { 2 - 3 } & $\mathrm{Z}=-2428155,6139$ & $\sigma_{Z}^{\text {ROSA }}=0,0017$ \\
\hline \multirow{4}{*}{ ILHA } & $\mathrm{X}=3785720,2377$ & $\sigma_{X}^{\text {OURI }}=0,0026$ \\
\cline { 2 - 3 } & $\mathrm{Y}=-4494897,6833$ & $\sigma_{Y}^{\text {OURI }}=0,0028$ \\
\cline { 2 - 3 } & $\mathrm{Z}=-2471710,4759$ & $\sigma_{Z}^{\text {OURI }}=0,0017$ \\
\hline & $\mathrm{X}=3735306,3504$ & $\sigma_{X}^{I L H A}=0,0030$ \\
\cline { 2 - 3 } & $\mathrm{Y}=-4669666,4224$ & $\sigma_{Y}^{I L H A}=0,0033$ \\
\cline { 2 - 3 } & $\mathrm{Z}=-2212269,3569$ & $\sigma_{Z}^{I L H A}=0,0020$ \\
\hline & $\mathrm{X}=3789545,4141$ & $\sigma_{X}^{S J R P}=0,0023$ \\
\cline { 2 - 3 } & $\mathrm{Y}=-4587255,7986$ & $\sigma_{Y}^{S J R P}=0,0026$ \\
\cline { 2 - 3 } & $\mathrm{Z}=-2290619,2435$ & $\sigma_{Z}^{S J R P}=0,0016$ \\
\hline
\end{tabular}

Para validação do ajustamento realiza-se o TGM (Teste Global do Modelo), com base na distribuição T-Student. Após realizar o ajustamento é possível calcular o fator de variância a posteriori pela seguinte equação 1 :

$$
\hat{\sigma}_{0}^{2}=\frac{v^{T} P v}{n-u}=\frac{3,180992417639}{30-15}=0,3428
$$

onde v: vetor dos resíduos;

$P$ : matriz dos pesos; e

$n-u$ : graus de liberdade.

No ajustamento, o fator de variância a priori utilizado foi de $\sigma_{0}^{2}=1$. Desta forma, aplica-se o TGM, utilizando $\alpha=5 \%$, para verificar se existem possíveis erros nas observações.

Portanto, formulam-se as hipóteses:

$$
\left\{\begin{array}{l}
H_{0}: E\left\{\hat{\sigma}_{0}^{2}\right\}=\sigma_{0}^{2} \\
H_{1}: E\left\{\hat{\sigma}_{0}^{2}\right\}>\sigma_{0}^{2}
\end{array}\right.
$$


Para a validação de uma das hipóteses, tem-se o seguinte valor calculado:

$$
T=\chi_{C}^{2}=\frac{\hat{\sigma}_{0}^{2}}{\sigma_{0}^{2}} \cdot(g . l)=5,1421
$$

A quantidade $T$ calculada tem distribuição $\chi^{2}$, com g.l (graus de liberdade)

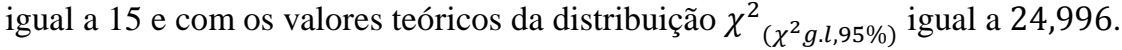

A hipótese nula $\left(H_{0}\right)$ não é rejeitada, ao nível de significância $\alpha=5 \%$, pois $T<\chi_{(g . l, 1-\alpha)}^{2}$.

\section{ANÁLISES DE CONFIABILIDADE}

O conceito de confiabilidade, segundo Kuang (1996), foi originado com Baarda (1968), e refere-se à capacidade de uma rede resistir a erros grosseiros nas observações. Existem dois tipos de medidas de confiabilidade, a interna e a externa. A primeira refere-se à capacidade de uma rede a permitir a detecção de pequenos erros nas observações com certo nível de confiança $(1-\alpha)$ e poder do teste $(1-\beta)$; e a segunda, quantifica a influência destas observações no cálculo das coordenadas dos pontos.

A prevenção de erros grosseiros deve ser realizada para evitar mascarar os resultados finais. Os testes globais de detecção possuem limites que às vezes não detectam magnitudes de erros que ainda persistem em algumas observações. Quando aplicado em redes, a confiabilidade tem dois significados: o primeiro está relacionado com a controlabilidade das observações da rede, que mostra o quão sensível a técnica de detecção de erros grosseiros é quando aplicado na rede; e a segunda refere-se principalmente ao quão sensível a rede é a estes resíduos de erros grosseiros que modificam as observações. Em outras palavras refere-se à robustez da rede, como a rede resiste às pequenas inconsistências nas observações em comparação a uma medida de precisão que relaciona apenas erros aleatórios (KUANG, 1996).

\subsection{Confiabilidade Interna}

Para encontrar a magnitude do menor erro detectável, investigam-se as implicações da aceitação da hipótese nula, no teste global do modelo, ou do QuiQuadrado, após o ajustamento. Como consequência desta aceitação, comete-se um erro do tipo II, ou seja, aceitar a hipótese nula quando ela não é verdadeira. Por meio desta teoria, pode-se estimar o valor mínimo de um erro grosseiro na observação que pode ser detectado (OLIVEIRA \& DALMOLIN, 2008).

Este valor mínimo $\left(\nabla_{0} l_{i}\right)$ pode ser localizado com níveis de probabilidade $\alpha_{0} \mathrm{e}$ $\beta_{0}$, calculado por: 
Cavalheri, E. P; Chaves, J. C.

$$
\nabla_{0} l_{i}=\sigma_{l i} \frac{\lambda_{0}}{\sqrt{r_{i}}},(i=1, \ldots, n)
$$

onde:

$\lambda_{0}$ : parâmetro de não centralidade;

$\sigma_{l i}$ : desvio-padrão da i-ésima observação;

$r_{i}$ : redundância parcial; e

$n$ : número de pontos.

Portanto, os erros mínimos não detectáveis são mostrados na Tabela 2.

Tabela 2 - Erros mínimos não detectados.

\begin{tabular}{|c|c|c|c|c|}
\hline \multirow{2}{*}{$\begin{array}{c}\text { Estações } \\
\text { de } \\
\text { referência }\end{array}$} & \multirow[t]{2}{*}{$\begin{array}{c}\text { Estações } \\
\text { investigadas }\end{array}$} & \multicolumn{3}{|c|}{$\begin{array}{c}\nabla_{0} l_{i}\left(\alpha_{0}=5 \%, \beta_{0}=20 \%, \lambda_{0}=\right. \\
2,80)\end{array}$} \\
\hline & & $\begin{array}{c}\nabla_{0} l_{i}(m) \\
\mathrm{em} \mathrm{X}\end{array}$ & $\begin{array}{c}\nabla_{0} l_{i}(m) \\
\mathrm{em} \mathrm{Y}\end{array}$ & $\begin{array}{c}\nabla_{0} l_{i}(m) \\
\mathrm{em} \mathrm{Z}\end{array}$ \\
\hline SJRP & \multirow[t]{2}{*}{ PPTE } & 0,0252 & 0,0286 & 0,0176 \\
\hline UFPR & & 0,0252 & 0,0286 & 0,0176 \\
\hline SJRP & \multirow[t]{2}{*}{$\overline{\text { ROSA }}$} & 0,0245 & 0,0280 & 0,0168 \\
\hline UFPR & & 0,0245 & 0,0280 & 0,0168 \\
\hline SJRP & \multirow[t]{2}{*}{ OURI } & 0,0246 & 0,0269 & 0,0170 \\
\hline UFPR & & 0,0246 & 0,0269 & 0,0170 \\
\hline SJRP & \multirow[t]{2}{*}{ ILHA } & 0,0273 & 0,0312 & 0,0180 \\
\hline UFPR & & 0,0273 & 0,0312 & 0,0180 \\
\hline SJRP & \multirow[t]{2}{*}{ SPAR } & 0,0222 & 0,0247 & 0,0151 \\
\hline UFPR & & 0,0222 & 0,0247 & 0,0151 \\
\hline
\end{tabular}

\subsection{Confiabilidade Externa}

Após aplicar a confiabilidade interna e encontrar o erro mínimo $\left(\nabla_{0} l_{i}\right)$, com base nas observações, torna-se necessário saber como os parâmetros responderão a estes erros. Assim, confiabilidade externa é definida como a quantidade em que o menor erro encontrado na observação pode influenciar no cálculo das coordenadas dos pontos.

A estimativa das coordenadas dos pontos, com base no ajustamento de observações no método paramétrico, é fornecida por:

$$
\mathrm{X}=-\left(A^{T} P A\right)^{-1} \cdot A^{T} P L
$$

Supondo que as equações contenham erros grosseiros não detectados $\left(\nabla_{0} l\right)$, reescreve-se a equação (5) da seguinte forma: 


$$
\begin{gathered}
X=-\left(A^{T} P A\right)^{-1} \cdot A^{T} P\left(L-\nabla_{0} l\right) \\
\mathrm{X}=-\left(A^{T} P A\right)^{-1} \cdot A^{T} P L+\left(A^{T} P A\right)^{-1} \cdot A^{T} P\left(l_{i} \nabla_{0} l\right)
\end{gathered}
$$

onde $\mathrm{l}_{\mathrm{i}}$ corresponde a i-ésima coluna de uma matriz identidade nxn.

Portanto, a influência dos erros grosseiros não detectados $\left(\nabla_{0} l_{i}\right)$ na estimativa das coordenadas é dada por:

$$
\nabla \mathrm{X}=\left(A^{T} P A\right)^{-1} \cdot A^{T} P\left(\nabla_{0} l\right) .
$$

Então, tem-se a confiabilidade externa, conforme Tabela 3.

Tabela 3 - Influência dos erros mínimos nas coordenadas.

\begin{tabular}{c|c|c|c}
\hline $\begin{array}{c}\text { Estações } \\
\text { investigadas }\end{array}$ & \multicolumn{3}{|c}{ Influência de $\left(\nabla_{\mathbf{0}} \boldsymbol{l}\right)$ nas coordenadas } \\
\hline & $\Delta \mathrm{X}(m)$ & $\Delta \mathrm{Y}(m)$ & $\Delta \mathrm{Z}(\mathrm{m})$ \\
\hline PPTE & 0,0252 & 0,0286 & 0,0175 \\
\hline ROSA & 0,0245 & 0,0280 & 0,0167 \\
\hline OURI & 0,0246 & 0,0269 & 0,0169 \\
\hline ILHA & 0,0273 & 0,0312 & 0,0179 \\
\hline SJRP & 0,0222 & 0,0246 & 0,0151 \\
\hline
\end{tabular}

\section{ANÁLISES DE DEFORMAÇÃO}

Como visto, a técnica de robustez analisa, com base nos erros máximos não detectáveis, a robustez de uma rede. Estes erros causam deslocamentos nas coordenadas dos pontos, sendo estes descritos como uma função de primeiro grau em cada uma das componentes, conforme as seguintes expressões:

$$
\begin{gathered}
u_{i}=u(x, y, z)=a_{0}+\frac{\partial u_{i}}{\partial x}\left(X_{j}-X_{i}\right)+\frac{\partial u_{i}}{\partial y}\left(Y_{j}-Y_{i}\right)+\frac{\partial u_{i}}{\partial z}\left(Z_{j}-Z_{i}\right), \\
v_{i}=v(x, y, z)=b_{0}+\frac{\partial v_{i}}{\partial x}\left(X_{j}-X_{i}\right)+\frac{\partial v_{i}}{\partial y}\left(Y_{j}-Y_{i}\right)+\frac{\partial v_{i}}{\partial z}\left(Z_{j}-Z_{i}\right), \\
w_{i}=w(x, y, z)=c_{0}+\frac{\partial w_{i}}{\partial x}\left(X_{j}-X_{i}\right)+\frac{\partial w_{i}}{\partial y}\left(Y_{j}-Y_{i}\right)+\frac{\partial w_{i}}{\partial z}\left(Z_{j}-Z_{i}\right) .
\end{gathered}
$$

Os coeficientes de cada equação correspondem aos parâmetros de deformação. Em forma matricial, as derivadas dos deslocamentos em função das coordenadas, constituem o tensor de deformação: 


$$
\mathrm{E}=\left[\begin{array}{lll}
\frac{\partial u_{i}}{\partial x} & \frac{\partial u_{i}}{\partial y} & \frac{\partial u_{i}}{\partial z} \\
\frac{\partial v_{i}}{\partial x} & \frac{\partial v_{i}}{\partial y} & \frac{\partial v_{i}}{\partial z} \\
\frac{\partial w_{i}}{\partial x} & \frac{\partial w_{i}}{\partial y} & \frac{\partial w_{i}}{\partial z}
\end{array}\right]
$$

Em função dos parâmetros de deformação, pode-se obter o deslocamento qualquer ponto da rede. Porém, antes, torna-se necessário calcular as condições iniciais $X_{0}, Y_{0}$ e $Z_{0}$. Como a equação (9) forma um sistema de equações diferenciais e de primeira ordem, a norma do vetor deslocamento de todos os pontos da rede é minimizada. O processo de integração para resolvê-las é explicado em detalhes em Vanìcek e Kimbere (1988 apud BERBER, 2006). Basicamente, a condição dirá onde a rede estava localizada antes da deformação.

Desta forma, calculam-se os deslocamentos individuais de cada ponto na rede com a equação (11).

$$
\left[\begin{array}{c}
u_{i} \\
v_{i} \\
w_{i}
\end{array}\right]=\left[\begin{array}{lll}
\frac{\partial u_{i}}{\partial x} & \frac{\partial u_{i}}{\partial y} & \frac{\partial u_{i}}{\partial z} \\
\frac{\partial v_{i}}{\partial x} & \frac{\partial v_{i}}{\partial y} & \frac{\partial v_{i}}{\partial z} \\
\frac{\partial w_{i}}{\partial x} & \frac{\partial w_{i}}{\partial y} & \frac{\partial w_{i}}{\partial z}
\end{array}\right] \cdot\left[\begin{array}{c}
X_{i}-X_{0} \\
Y_{i}-Y_{0} \\
Z_{i}-Z_{0}
\end{array}\right]
$$

Após o cálculo das componentes do deslocamento de cada ponto, pode-se calcular a quantidade total de deslocamento, obtidos em função das análises de confiabilidade e deformação, conforme a equação (12).

$$
D_{i}=\sqrt{u_{i}^{2}+v_{i}^{2}+w_{i}^{2}} .
$$

\subsection{Valores Limites}

No ajustamento das linhas de bases, obtiveram-se as coordenadas e a MVC (matriz covariância) das posições. Com base na elipse padrão de erros é possível obter uma interpretação visual da acurácia dos pontos. Esta elipse é obtida pelos seus semi-eixos maior $(a)$ e menor $(b)$, que utilizam os elementos da MVC dos parâmetros para a sua obtenção, conforme as seguintes fórmulas:

$$
\begin{gathered}
\sigma_{x^{\prime}}^{2}=0,5\left({\sigma_{x}}^{2}+{\sigma_{y}}^{2}\right)+0,5 M \\
\sigma_{y^{\prime}}^{2}=0,5\left({\sigma_{x}}^{2}+{\sigma_{y}}^{2}\right)-0,5 M
\end{gathered}
$$




$$
M=\sqrt{4 \sigma_{x y}^{2}+\left(\sigma_{x}^{2}-\sigma_{y}^{2}\right)^{2}} .
$$

Os valores $\sigma_{x}^{2}$ e $\sigma_{y^{\prime}}^{2}$ proporcionam os semi-eixos maior e menor da elipse de erros:

$$
\begin{aligned}
& a=\sqrt{\sigma_{x}^{2}} \\
& b=\sqrt{\sigma_{y^{\prime}}^{2}}
\end{aligned}
$$

A elipse obtida com $a$ e $b$ é denominada de elipse dos erros padrão, e tem $39 \%$ de probabilidade de que o ponto esteja dentro dela.

A elipse de confiança é obtida multiplicando as quantidades $\sigma_{x}^{2}$, e $\sigma_{y}^{2}$ por um fator $K$, conforme Tabela 4 .

Tabela 4 - Níveis de probabilidade da elipse dos erros padrão.

\begin{tabular}{c|cc}
\hline Probabilidade & Distribuição $\chi_{(2 \text { g.l) }}^{2}$ & $K=\sqrt{\chi_{(2 \text { g.l })}^{2}}$ \\
\hline $99,5 \%$ & 10,5966 & 3,26 \\
\hline $95 \%$ & 5,991 & 2,45 \\
\hline $90 \%$ & 4,605 & 2,15 \\
\hline $70 \%$ & 2,409 & 1,55 \\
\hline $50 \%$ & 1,3863 & 1,18 \\
\hline
\end{tabular}

Por exemplo, a elipse padrão com $95 \%$ de probabilidade é dada por:

$$
\begin{aligned}
& a_{95 \%}=K \cdot \sigma_{x^{\prime}}^{2}=2,45 \cdot \sigma_{x^{\prime}}^{2} \\
& b_{95 \%}=K \cdot \sigma_{y^{\prime}}^{2}=2,45 \cdot \sigma_{y^{\prime}}^{2}
\end{aligned}
$$

Para obter o intervalo de confiança que representará a terceira componente $\left(h_{95 \%}\right)$, multiplica-se $\sigma_{h}$, extraído da matriz covariância, por um fator de expansão da raiz quadrada de uma Qui-Quadrado com um grau de liberdade $\chi_{(1 \mathrm{~g} . l)}^{2}$. Portanto, para um intervalo de confiança de $95 \%$, tem-se:

$$
h_{95 \%}=\sqrt{\chi_{(1 \mathrm{~g} . l)}^{2}} \cdot \sigma_{h}=1,96 \cdot \sigma_{h}
$$

Desta forma, os valores limites, para cada ponto, são: 


$$
\delta_{i}=\sqrt{a_{95 \%}^{2}+b_{95 \%}^{2}+h_{95 \%}^{2}}
$$

\subsection{Análise de Robustez}

Realiza-se a análise de robustez com a comparação dos deslocamentos calculados pelas equações (12) e (21). Se para um dado ponto $P_{i}, D_{i}>\delta_{i}$, diz-se que a rede neste local é fraca, ou seja, não é "robusta". Se $D_{i}<\delta_{i}$, a rede neste local é dita "robusta" a certo nível de probabilidade.

$\mathrm{Na}$ Tabela 5, verifica-se a análise da robustez de cada ponto da rede investigada.

Tabela 5 - Análise da robustez de cada ponto da rede investigada.

\begin{tabular}{l|c|c|c}
\hline Pontos & $\boldsymbol{D}_{\boldsymbol{i}}(\boldsymbol{m})$ & $\boldsymbol{\delta}_{\boldsymbol{i}}(\boldsymbol{m})$ & Análise da Robustez \\
\hline PPTE & 0,0121 & 0,0104 & Não robusto \\
\hline ROSA & 0,0094 & 0,0101 & Robusto \\
\hline OURI & 0,0257 & 0,0100 & Não robusto \\
\hline ILHA & 0,0097 & 0,0112 & Robusto \\
\hline SPAR & 0,0029 & 0,0090 & Robusto \\
\hline
\end{tabular}

Observa-se que as estações classificadas como não robustas são PPTE e OURI, com valores do deslocamento devido aos erros grosseiros de $1,2 \mathrm{~cm}$ e $2,6 \mathrm{~cm}$, respectivamente. As estações ROSA, ILHA e SPAR têm deslocamentos menores que os limiares de aceitação, portanto a rede é robusta na região de abrangência destas estações.

\section{DISCUSSÕES E CONCLUSÕES}

Para execução da técnica de análise de robustez é necessário determinar deslocamentos sofridos pelos pontos em função do erro máximo não detectável, obtido pelas análises de confiabilidade interna e externa. Antes de calcular estes deslocamentos, foi necessário determinar condições iniciais, na qual diz onde a rede estava antes da deformação. Posteriormente determinam-se os limiares que irão classificar pontos robustos ou não. Estes limiares consideram somente a acurácia das observações.

Pelos cálculos efetuados, alguns pontos foram classificados como robustos e, em outros pontos, como não robustos. Dos cinco pontos analisados, três foram classificados como robustos, e o restante, dois pontos, como não robustos. Nos locais robustos, entende-se que em função da geometria da rede e acurácia das observações, a rede resistiu aos erros grosseiros. Já nos pontos onde a rede não foi 
classificada como robusta, a geometria foi inadequada, ou, os pontos não tiveram redundância, ou seja, as observações não foram suficientes. Quando um ponto ou certa região da rede não são classificados como robustos, sugere-se que esta tenha uma mudança na sua geometria, ou seja, a rede deverá ser redesenhada. Também, aumentam-se as observações entre os pontos até atingir graus de robustez aceitáveis.

As técnicas estatísticas de análises de erros, após o ajustamento, às vezes não são capazes de encontrar alguns erros grosseiros nas observações. Assim a análise de robustez é uma ferramenta uma vez que vem para suprir esta deficiência dos testes. Ressaltando de que a análise não depende do datum, dependendo somente da geometria e acurácia das observações.

\section{AGRADECIMENTOS}

Agradecemos à FAPESP pela bolsa de iniciação científica, processo $\mathrm{n}^{\circ}$ 2010/09255-3 cedida, sem a qual não seria possível o andamento e conclusão deste projeto. Também ficamos gratos ao MIT pela disponibilização dos programas GAMIT e GLOBK para fins científicos.

\section{REFERÊNCIAS BIBLIOGRÁFICAS}

BAARDA, W. A testing procedures for use in geodetic networks. Netherlands Geodetic Commission, Publications on Geodesy, New Series, Vol. 2, No 5, Delft, Netherlands, 1968.

BERBER, M. Robustness analysis of geodetic networks. Ph.D. Dissertation, department of geodesy and geomatics engineering, technical report no. 242, University of New Brunswick, Fredericton, new Brunswick, Canada, 2006.

HERRING, T. A.; KING, R. W.; McCLUSKY, S. C. Introduction to GAMIT/GLOBK. department of Earth, Atmospheric, and Planetary Sciences. Massachusetts Institute of Technology. Release 10.4, October 2010.

GAMIT Reference Manual: GPS analysis at MIT. Department of Earth, Atmospheric, and Planetary Sciences. Massachusetts Institute of Technology. Release 10.4, October 2010. GLOBK Reference Manual: Global Kalman filter VLBI and GPS analysis program. Department of Earth, Atmospheric, and Planetary Sciences. Massachusetts Institute of Technology. Release 10.4, October 2010.

KUANG, S. Geodetic network analysis and optimal design: concepts and applications. Ann Arbor Press, Inc. Chelsea, Michigan, USA, 1996.

OLIVEIRA, R.; DALMOLIN, Q. A influência da Redundância da Observação sobre a Precisão dos Parâmetros. Curso de Pós-Graduação em Ciências Geodésicas, Departamento de Geomática, Universidade Federal do Paraná. Boletim de Ciências Geodésicas, Vol. 14, n. 3, p.295-315. Curitiba, Paraná, 2008. 
VANİCEK, P.; KRAKIWSKY, E.J; CRAYMER M.R. Robustness analysis of geodetic horizontal networks. Journal of geodesy, 75,199-209, 2001.

(Recebido em outubro de 2013. Aceito em fevereiro de 2014). 